\title{
p53 and mitochondrial dysfunction: novel insight of neurodegenerative diseases
}

\author{
Chun-Qiu Dai ${ }^{1}$ - Ting-Ting Luo ${ }^{2}$ Shi-Cheng Luo ${ }^{1} \cdot$ Jia-Qi Wang ${ }^{1}$. \\ Sheng-Ming Wang ${ }^{1} \cdot$ Yun-Hu Bai $^{3} \cdot$ Yan-Ling Yang $^{3} \cdot$ Ya-Yun Wang ${ }^{1}$
}

Received: 22 April 2016 / Accepted: 7 July 2016 / Published online: 15 July 2016

(C) Springer Science+Business Media New York 2016

\begin{abstract}
Mitochondria are organelles responsible for vital cell functions. p53 is a transcription factor that regulates the DNA stability and cell growth normality. Recent studies revealed that $\mathrm{p} 53$ can influence mitochondrial function changing from normal condition to abnormal condition under different stress levels. In normal state, p53 can maintain mitochondrial respiration through transactivation of $\mathrm{SCO} 2$. When stress stimuli presents, $\mathrm{SCO} 2$ overexpresses and leads to ROS generation. ROS promotes p53 inducing MALM (Mieap-induced accumulation of lysosome-like organelles within mitochondria) to repair dysfunctional mitochondria and MIV (Mieap-induced vacuole) to accomplish damaged mitochondria degradation. If stress or damage is irreversible, p53 will translocate to mitochondria, leading into apoptosis or necrosis. Neurodegenerative diseases including Parkinson's disease, Huntington's disease and Alzheimer's disease are still lack of clear explanations of mechanisms, but more studies have revealed the functional relationship between mitochondria and p53 towards the pathological development of these diseases.
\end{abstract}

Chun-Qiu Dai and Ting-Ting Luo contributed equally to this study.

Yan-Ling Yang and Ya-Yun Wang These authors contributed equally to this study and share the corresponding authorship.

Ya-Yun Wang

Wang_YaYun_fmmu@163.com

1 Department of Anatomy, Histology and Embryology, K.K. Leung Brain Research Centre, The Fourth Military Medical University, Xi'an 710032, China

2 Department of Neurobiology and Collaborative Innovation Center for Brain Science, School of Basic Medicine, The Fourth Military Medical University, Xi'an 710032, China

3 Department of Hepatobiliary Surgery, Xi-Jing Hospital, The Fourth Military Medical University, Xi'an 710032, China
In this review, we discuss that p53 plays the vital role in the function of mitochondria in the aspect of pathological change metabolism. We also analyze these diseases with novel targeted treating molecules which are related to p53 and mitochondria, hoping to present novel therapies in future clinic.

Keywords Mitochondria · p53 · Mitochondrial quality control · Apoptosis · Mitochondrial dysfunction · Neurodegenerative diseases $\cdot$ Molecular therapy

\section{Introduction}

Neurodegenerative diseases are common but hard to cure. At the same time, the mechanisms still need further study. Fortunately, recent studies have revealed some relationships between mitochondria and p53 in the development of neurodegenerative diseases. Mitochondria are organelles that are responsible for several vital cell functions, including respiration, oxidative phosphorylation, and regulation of apoptosis. Moreover, they are also the main intracellular place for generating reactive oxygen species (ROS) (Gibellini et al. 2015). p53 protein is a transcription factor that regulates the DNA stability and cell growth normality. It can maintain the DNA stability by inhibiting its mutation. Stresses like DNA damage will turn on its function, thus, leading to cell cycle arrest for DNA repair, senescence, cell growth arrest, and therefore, apoptosis (Chen et al. 2010).

However, the mechanism of mitochondrial and p53 pathway towards the neurodegenerative diseases haven't been clarified clearly.

Further studies have revealed that p53 can destruct the regulation of mitochondrial function in stress state, which contributes to the abnormal neuronal condition and the occurrence and development of some neuronal diseases. Based on these 
previous researches, we have some novel insights of the relationship between mitochondria and p53 especially in the aspect of neurodegenerative diseases, hoping to present novel molecular therapies in future clinic. In this review, we discuss the influence of p53 upon mitochondria shifting from normal condition to abnormal condition under different stress levels, as well as the relations of p53 and mitochondria to etiology and molecular therapies of three neurodegenerative diseases.

\section{p53 regulates the mitochondrial respiration}

In normal condition, p53 can modulate the utilization of mitochondrial respirator. An experiment reported that HCT116 p53+/+ cells have higher oxygen consumption than HCT116 p53-/- cells, suggesting that p53 can regulate mitochondrial respiratory activity (Bergeaud et al. 2013).

Cytochrome c oxidase (COX) is known as complex IV and plays the vital role in the respiratory chain. It contains three mitochondrial DNA encoding subunits and ten nuclear DNA encoding subunits (Assaily and Benchimol 2006, Yang et al. 2010).

Synthesis of Cytochrome c Oxidase 2 (SCO2) is one of the nuclear DNA encoding subunits, p53 can binds to the $\mathrm{SCO} 2$ promoter in nuclear DNA and regulating mitochondrial respiration. In the experiment of Satoaki Matoba et al., they used human cancer cells with wild-type p53 represents the p53deficient cell. The disruption of the $\mathrm{SCO} 2$ gene represent the metabolic switch towards glycolysis in the p53-deficient cell. The result shows that $\mathrm{p} 53$-deficient cell down regulates the mitochondrial respiration (Matoba et al. 2006), indicating the mitochondrial regulation by $\mathrm{p} 53$.

Moreover, Jerad Fields et al. demonstrated that $\mathrm{SCO} 2$ can rescue the decreased aerobic respiration in p53-deficient cells at physiologic levels, confirming that p53 can transactivate the SCO2 gene (Fig. 1b) (Fields et al. 2007).

However, this binding is not beneficial all the time. To keep the balance of the respiration and avoid the Warburg effect, the cells will promote $\mathrm{p} 53$ expression and thus, resulting the SCO2 overexpression (Madan et al. 2013), but ROS will also be generated during this procedure. Mitochondrial-generated ROS will induce p53 translocation to nuclear and mitochondria, leading into the Mieap expression and mitochondria mediated apoptosis (Qi et al. 2011).

\section{p53 involved mitochondrial quality control: MALM and MIV}

After stress stimuli, p53 will function as to control mitochondrial quality. Mitochondrial quality control (MQC) is significantly important for maintaining the stable and healthy state of our bodies. There are two possible mechanisms of the quality control. The first one is mitophagy, mediated by double- membraned autophagosomes. The second one is proteasedependent degradation of damaged proteins in mitochondria. Moreover, many novel studies confirmed the vital role of the interaction between $\mathrm{p} 53$ and mitochondria towards MQC.

\section{MALM and MIV}

Mieap, a p53-inducible protein, was found which has influence on the MQC in mouse intestinal tumor (Tsuneki et al. 2015) and human colorectal cancer (Kamino et al. 2016), leading to the repair of mitochondria function.

Accumulation of the oxidized protein was reported to impair the function of the mitochondria, leading to the mitochondrial dysfunction. Mieap repairs unhealthy mitochondria by eliminating the oxidized mitochondrial proteins, including ATP synthase b-subunit. Therefore, it maintains the quality of mitochondria (Miyamoto et al. 2011). Besides, Noriaki Kitamura et al. discovered two novel mechanisms, MALM (Mieap-induced accumulation of lysosome-like organelles within mitochondria) and MIV (Mieap-induced vacuole), and they both contribute to the process of MQC.

In MALM, Mieap-regulated lysosome in mitochondria is not similar to canonical autophagy. Destruction of the mitochondrial structure on electron microscopy and the loss of mitochondrial signal on immunofluorescence are the character in mitophagy, but these are not found in MALM (Miyamoto et al. 2011). Instead, MALM induces intramitochondrial lysosome-like organella without destroying the mitochondrial structure, thus, leading to the elimination of oxidized mitochondrial proteins and improvement of mitochondrial functions afterwards (Kitamura et al. 2011). During this procedure, NIX and BNIP3 were found to take the vital role in targeting and repairing the damaged mitochondria. NIX and BNIP3 are localized in outer mitochondria membrane, which will be activated by the ROS under the stress condition that can also activate Mieap expression. BH3 domain of activated NIX and BNIP3 can collect Mieap and interact with its coiled-coil domain. With the help of Mieap, NIX and BNIP3 can form a pore which allows the translocation of the combination of Mieap and lysosome from cytoplasm to mitochondrial matrix, leading into the degradation of oxidized protein (Kitamura et al. 2011). Moreover, another protein 14-3-3 $\gamma$, a member of the 14-3-3 family of proteins as well as Mieap-interacting protein is also demonstrated to take the vital role in MALM. Takafumi Miyamoto et al. found that $14-3-3 \gamma$ is translocated from the cytoplasm to mitochondrial matrix during MALM. Moreover, further study showed that $14-3-3 \gamma$ is involved in decreasing oxidized mitochondrial proteins, which helps to the function of MALM (Miyamoto et al. 2012) (Fig. 1c).

With enhancement of the stress level, MALM can be inhibited, MIV degrade the dysfunctional mitochondria by accumulating lysosomes. It is observed that lysosomes accumulate around the edge of a MIV and fuse to the MIV 


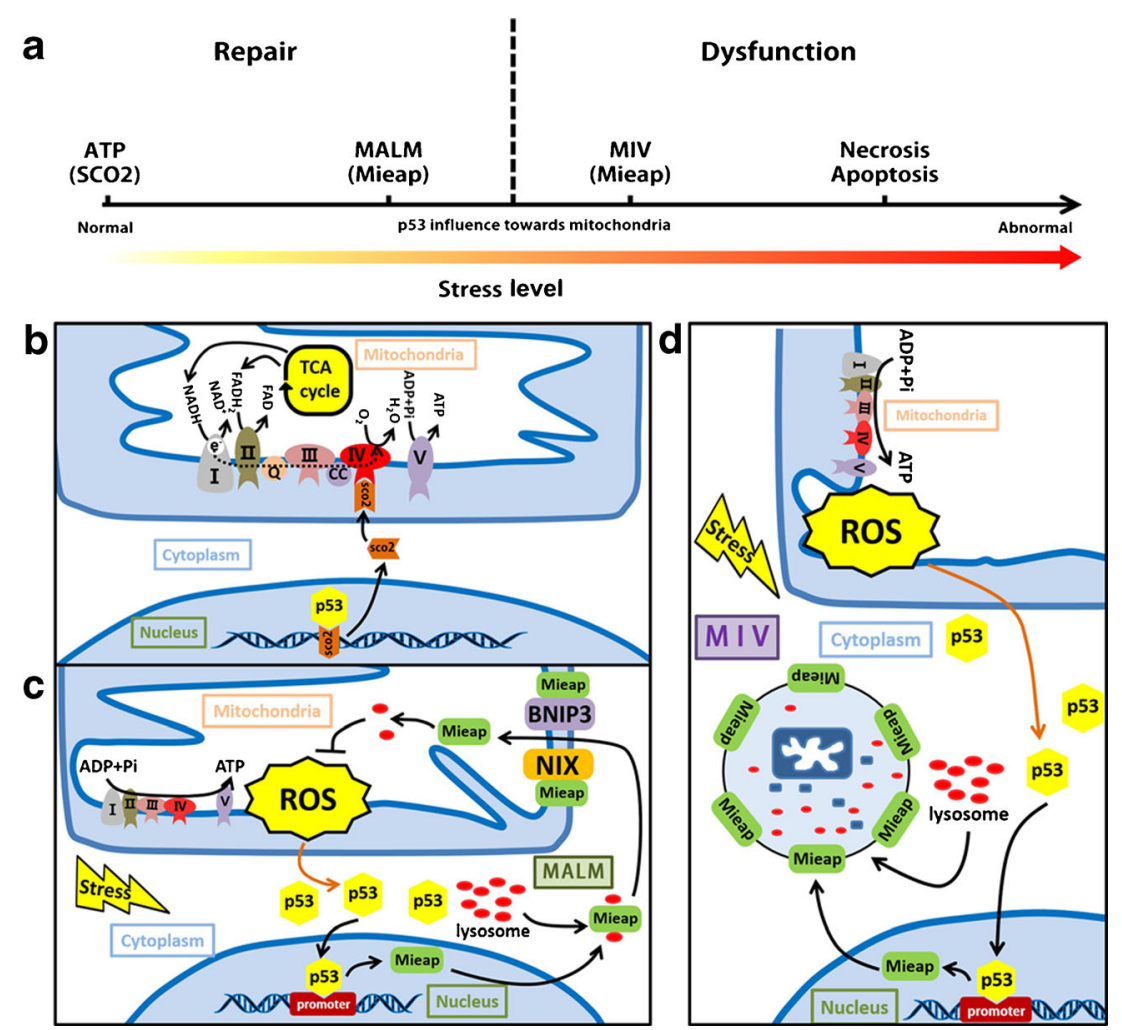

Fig. 1 p53 plays numerous roles in mitochondria-related processes. a p53 influence towards mitochondria under different stress level. With the enhancement of stress level, mitochondrial function will switch from normal to abnormal in the presence of $\mathrm{p} 53$. b p 53 regulation and transactivation of mitochondrial Synthesis of Cytochrome c Oxidase 2 (SCO2). The figure shown is the nuclear transactivation of $\mathrm{SCO} 2$ by $\mathrm{p} 53$. $\mathrm{SCO} 2$ is targeted to the inner membrane of the mitochondria where they bind to complex IV and promote aerobic respiratory. When the cell is under the primary stress state, the cells will promote $\mathrm{p} 53$ expression keep the balance of the respiration and avoid the Warburg effect and thus, resulting the $\mathrm{SCO} 2$ overexpression, but ROS will also be generated during this procedure. c Mieap-induced accumulation of lysosome-like organelles within mitochondria (MALM). Mieap is a p53 inducible protein. Mitochondrial-generated ROS will induce p53

membrane. When the lysosomes are abundant, MIV will lead to the degradation of the mitochondria. But the detailed molecular regulation of this mechanism needs further study.

In conclusion, MALM plays a role in repairing mitochondria, whereas MIV degrade mitochondria. MIV should be enhanced while MALM is inhibited to maintain the goal of controlling the mitochondrial quality (Kitamura et al. 2011). (Fig. 1d).

\section{Mitochondrial p53 translocation and its related apoptosis and necrosis}

When stress or damage is irreversible, p53 will translocate to mitochondria, leading into apoptosis, necrosis. translocation to nuclear and mitochondria. p53 binds to Mieap promoter and leads to the Mieap overexpression. Mieap will binds to NIX and BNIP3, causing the activity change of NIX and BNIP3. These two protein can form a mitochondrial transition-like pore, permitting the translocation of Mieap and lysosome complex into mitochondrial matrix, thus, degenerating ROS and other oxidative protein. d Mieap-induced vacuole (MIV). With the enhancement of stress level, MALM is inhibited, Mieap will form a vacuole and degenerate the impaired mitochondria. Abbreviations: SCO2: mitochondrial Synthesis of Cytochrome c Oxidase 2, MALM: Mieap-induced accumulation of lysosome-like organelles within mitochondria, MIV: Mieap-induced vacuole, ROS: reactive oxygen species, NIX: NIP-like protein X, BNIP3: BCL2/adenovirus E1B $19 \mathrm{kDa}$ protein-interacting protein 3

\section{Mitochondrial p53 translocation}

\section{The detrimental function of p53 translocation}

The translocation of mitochondrial p53 contributes to the cells metabolism changes including changes of mitochondrial membrane potential, oxidative stress, cytochrome c release, and mitophagy, which leads to mitochondrial dysfunction afterwards, and therefore, causing p53-induced apoptosis and necrosis.

\section{Factors to influence mitochondrial p53 translocation}

Many factors are found to influence the mitochondrial p53 translocation, including stress, exercise, mitochondrial uncoupling and DNA damage. 
Christine Sansomed et al. found that short term hypoxic stress induces mitochondrial p53 using isolated mitochondria shown by electron microscopy. Significant amount of mitochondrial p53 induced by stress is located at the surface of the organelle (Sansome et al. 2001).

Interestingly, acute exercise was demonstrated to reduce nuclear $\mathrm{p} 53$, but it enhances the mitochondrial abundance of $\mathrm{p} 53$. Moreover, endurance exercise can be the signal to localize p53 to the mitochondria (Saleem and Hood 2013).

DNA damage may lead to the translocation of mitochondrial p53 is revealed as well (Hernlund et al. 2009).

Besides, Fei Wang et al. uncoupled the oxidative phosphorylation by carrying protons across the mitochondrial membrane and detected the p53 of 12-O-tetradecanoylphorbol 13-acetate (TPA) treated cells. Result shows that mitochondrial uncoupling can block p53 translocation (Wang et al. 2010).

\section{Regulation of mitochondrial p53 translocation}

Among these factors, we highlight the related molecules in stress state and its pathway of the procedure in mitochondrial p53 translocation.

It has already been mentioned that cytoplasmic p 53 rapidly translocate to mitochondria in response to a stress signal. This direct translocation of $\mathrm{p} 53$ results in robust mitochondrial outer membrane permeabilizing and causing apoptosis. According to the influence towards apoptosis, proteins on mitochondria are classified into anti-apoptotic and proapoptotic protein. Pro-apoptotic protein contain Bax、Bak、tBid、Bim 、 Puma and Noxa while antiapoptotic protein include $\mathrm{Bcl}-2$ family members. In normal state, Bcl-2 family members combine with Bax and Bak, inhibiting the apoptotic function of these two proteins. When stress stimuli presents, $\mathrm{p} 53$ will translocate into mitochondrial outer membrane and binds to $\mathrm{Bcl}-2$ or Bcl-xL, leading into the release of Bax and Bak. Bax and Bak can form the permeability pore respectively, which mediate the mitochondrial dysfunction through the formation of mitochondrial permeability transition pore (MPTP). Anti-apoptotic and pro-apoptotic proteins interact and localize on the mitochondrial membrane to keep the balanced state.

After the stress stimuli, mitochondrial p53 accumulation can be detected (Erster et al. 2004). Nuclear p53 transactivates its target gene Puma and induce the formation of Puma/Bcl-xL complex, which could liberate p53 in cytoplasm. In stress state, monoubiquitinated p53 can change into polyubiquitination state with the help of Murine Double Minute 2 (MDM2). Polyubiquitinated p53 can switch to deubiquitination state and translocate to the outer mitochondrial membrane, neutralizing the inhibitory function of Bcl-2/ $\mathrm{xL}$ in the presence of HAUSP (Herpesvirus Associated Ubiquitin Specific Protease). Bax and Bak can then be released and form the pores, leading to the cytochrome $\mathrm{c}$ release.
Besides, deubiquitination p53 can interact inner membrane potential. Complex of cyclophilin D and p53 on inner membrane contribute the permeabilization as well (Fig. 2) (Vaseva and Moll 2009).

\section{p53 involved apoptosis}

As discussed previously, the translocation of p53 may lead to mitochondrial dysfunction. This change leads to apoptosis eventually. After stress stimuli, p53 induces MPTP on both outer and inner membranes. The outer membrane MPTP can induce cytochrome c release, which is the core factor of apoptosis.

Besides MPTP, other factors are found to induce apoptosis as well. Jie Feng et al. found that PUMA (p53 upregulated modulator of apoptosis) is required for neuronal apoptosis (Feng et al. 2015). An experiment records that the suppression of p53 effectively mitigates disease-associated mitochondrial and dysfunction, thus, decrease the apoptosis (Wang et al. 2014). Besides, NOXA (a kind of apoptosis regulating factor) was found to mediate the p53-dependent apoptosis through mitochondrial dysfunction as well. Young-Woo Seo et al. indicated that NOXA seems to cause changes like activating the permeability transition-related pore, thus, releasing cytochrome c from mitochondria into cytosol (Seo et al. 2003). Ji Young Le et al. demonstrated that in the presence of p53, 2methoxyestradiol (2-MeO-E2) may cause cellular changes, like reducing the cell viability and enhancing the proportions of $\mathrm{G} 2 / \mathrm{M}$ cells and apoptotic sub-G1 cells, which may induce the apoptosis (Lee et al. 2014). Moreover, p53 is still be demonstrated as the vital factor in Mn-induced neuronal apoptosis (Wan et al. 2014).

Moreover, we present some anti-apoptotic molecules towards the changes. Chun-hua Wan et al. demonstrated that blockage of p53 activities with Pifithrin- $\alpha$ (PFT- $\alpha)$ and Pifithrin- $\mu$ (PFT- $\mu)$ can reduce Mn-induced mitochondrial $\mathrm{H}_{2} \mathrm{O}_{2}$ production, and the pretreatment with PFT- $\alpha$ and PFT $\mu$ has significantly weaken Mn-induced apoptosis in PC12 cells (Wan et al. 2014). High-mobility group A1 protein (HMGA1) is also reported as an inhibition factor. F Esposito et al. found that with counteracting the binding of $\mathrm{p} 53$ to the anti-apoptotic fact or Bcl-2, HMGA1 could inhibit p53mitochondrial apoptosis (Esposito et al. 2012). Moreover, SANG KYU LEE et al. demonstrated that phosphorylation of eIF $\alpha$ (eukaryotic translation initiation factors) can inhibit the stabilization and translocation of p53 to the mitochondria, and therefore, decreasing statin-induced apoptosis (Lee and Kim 2013).

\section{Mitochondria-related necrosis}

p53 can regulate apoptosis. In addition, it can also regulate necrotic cell death via the mitochondria. p53-mediated 
a

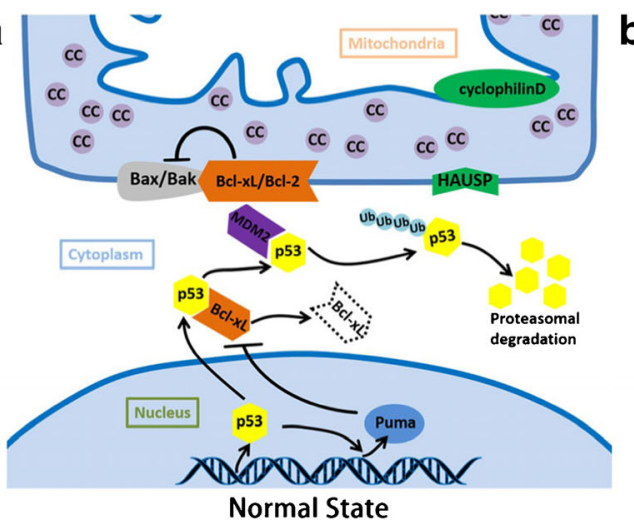

Fig. 2 p53 induced apoptosis and necrosis. a The molecules and pathway in mitochondrial p53 translocation in normal state. After translation, Bcl-xL will bind to $\mathrm{p} 53$ and inhibits its function. Nuclear p53 transactivates its target gene Puma and induce the formation of Puma/Bcl-xL complex, which could liberate p53 in cytoplasm. MdM2 contributes to the monoubiquitination of $\mathrm{p} 53$, leading into the $\mathrm{p} 53$ degradation. b The molecules and pathway in mitochondrial p53 translocation in stress state. After the stress stimuli, mitochondrial p53 accumulation can be detected. Monoubiquitinated p53 can change into polyubiquitination state. Polyubiquitinated p53 can switch to deubiquitination state and translocate to the outer mitochondrial membrane, neutralizing the inhibitory function of $\mathrm{Bcl}-2 / \mathrm{Bcl}-\mathrm{xL}$ in the

regulation of necrosis is associated with inner membrane MPTP control by interacting with cyclophilin D (CypD) and dynamin-related protein 1(Drp1). CypD is connected with MPTP opening related to oxidative stress (Dashzeveg and Yoshida 2015). p53 can accumulate in the inner mitochondrial membrane, and form complexes with cyclophilin D (CypD). This complex can mediate the inner membrane MPTP opening.

Drp1 is a large GTPase which can control the primary mitochondrial fission. Drp1 localizes to the cytoplasm. When the cell is under the condition of oxidative stress, it can bind to p53, stabilize it and play the vital role in p53 translocation to the mitochondria. Xing Guo et al. found that Drp1 binds to p53, thus, inducing mitochondria-related necrosis. They used wild-type Drp1 and Drp1 knockout mouse embryonic fibroblasts (MEFs) and demonstrated that Drp1 stabilizes p53 and helps to accumulate p53 on mitochondria, especially under condition of oxidative stress (Guo et al. 2014).

Nurmaa Dashzeveg et al. further identified that $\mathrm{p} 53$ binds to the N-terminal GTPase domain of Drp1, and Drp1 is required to assist translocation of p53 into mitochondria (Dashzeveg and Yoshida 2015).

During the study of Drp1, a novel molecule p110 is found which can inhibit Drp1 and p53. When treated with p110, association of Drp1 and p53 on mitochondria were inhibited in rats. In the experiment, p110 treatment can reduce brain infarction in rats. And p110 can be a good molecular targeted therapy of Huntington's disease. Details are discussed in the following part (Guo et al. 2014).

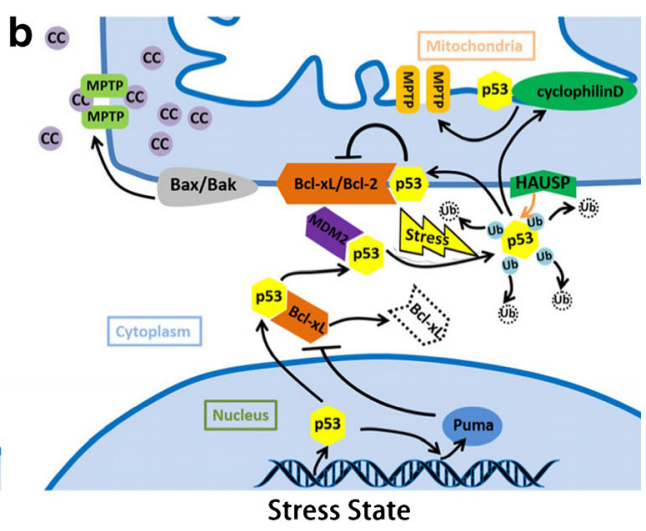

presence of HAUSP. Bax and Bak can then be released and form the MPTP, leading to the cytochrome c release, and apoptosis finally. Besides, deubiquitination p53 can decrease inner membrane potential. p53 can bind to cyclophilin D on inner membrane, activated cyclophilin D contributes the inner membrane MPTP as well, leading into the necrosis finally. Abbreviations: cc: cytochrome c, MPTP: mitochondrial permeability transition pore. HAUSP: herpesvirus associated ubiquitin specific protease, Bcl-2: B-cell lymphoma 2, Bcl-xL: B-cell lymphomaextra-large (BCL2-like 1), Bak: Bcl-2-homologous antagonist/killer, Bax: Bcl-2-associated X protein, Puma: p53 upregulated modulator of apoptosis, MDM2: Murine Double Minute 2

\section{Novel insight of typical neurodegenerative diseases}

Further studies reveal that $\mathrm{p} 53$ can destruct the regulation of mitochondrial function, which contributes the abnormal neuronal condition and the occurrence and development of some neurodegenerative diseases. Based on these novel mechanisms, we discuss typical neuronal diseases upon novel insight of the relationship between mitochondria and p53, hoping to present novel therapies towards these diseases.

\section{Parkinson's disease}

It is now generally accepted that Parkinson's disease (PD) is associated with a selective loss of dopamine (DA) neurons, especially in the substantia nigra of the midbrain (Park et al. 2015). Recent studies showed that mitochondrial dysfunction can results in energy crises and therefore, progressive dopaminergic neuronal apoptosis, which is the vital cause of PD (Hang et al. 2015).

We summarize two mainly functions of p53 and mitochondria in the development of PD. As we discussed previously, p53 can bind to Bcl-2 and release the Bax/Bak to form the MPTP, leading into the apoptosis eventually. Moreover, Denis Ottolini et al. found out that overexpression of p53 can drastically reduce mitochondrial $\mathrm{Ca} 2+$ transients (recombination of $\mathrm{Ca} 2+$ and sensitive photoprotein aequorin) in stimulated cells and permit more $\mathrm{Ca} 2+$ released into mitochondria matrix, thus, damaging mitochondrial morphology and causing mitochondrial dysfunction. (Ottolini et al. 2013). This 


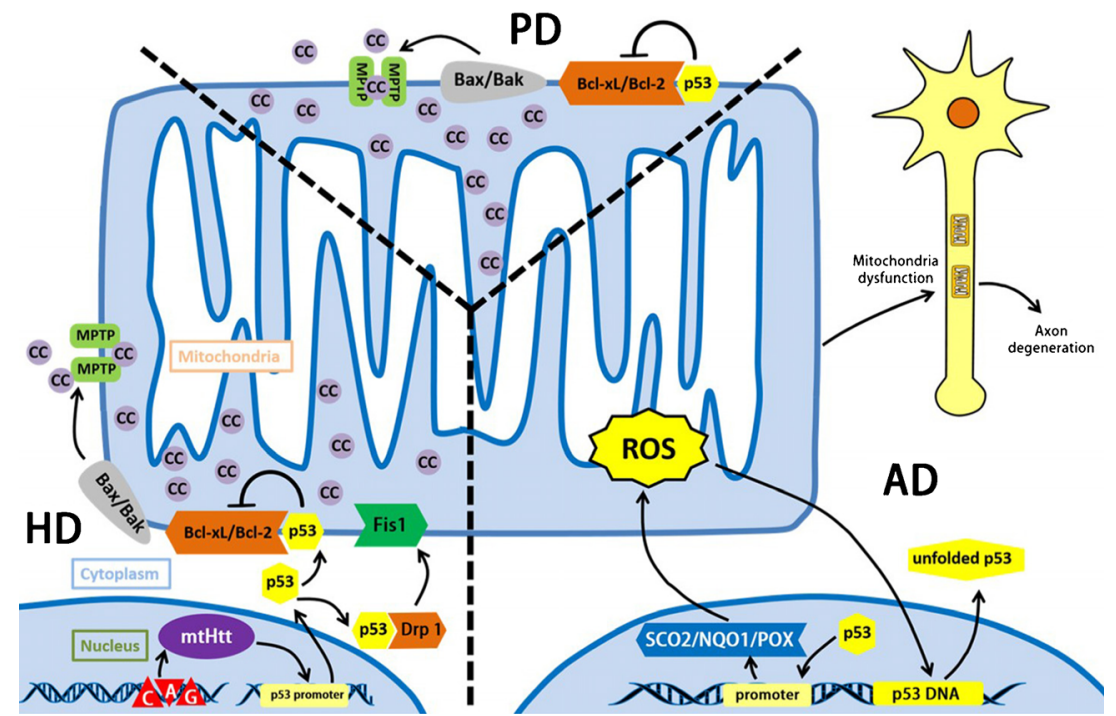

Fig. 3 p53 and mitochondria related etiology of three neurodegenerative diseases. Parkinson's disease (PD) is now generally accepted that PD is associated with a selective loss of dopamine (DA) neurons, especially in the substantia nigra of the midbrain, p53 can bind to Bcl-2/Bcl-xL and release the $\mathrm{Bax} / \mathrm{Bak}$ to form the MPTP, leading into the apoptosis finally. Huntington's disease (HD) is caused by the expansion of the coding CAG repeat in the open reading frame of the Huntingtin gene. The result of accumulation and expression of mutated Huntingtin protein $(\mathrm{mtHtt})$ will lead to cellular dysfunctions, thus, causing HD. mtHtt binds to p53 and upregulates nuclear p53 level as well as p53 transcriptional activity. With the help of $\mathrm{mtHtt}, \mathrm{p} 53$ binds to activated Drp1 under the stress state, which greatly enhance the interaction of Drp 1 and Fis 1 on mitochondrial outer membrane, causing the mitochondrial fission. Besides, the interaction of $\mathrm{Bcl}-\mathrm{xL} / \mathrm{Bcl}-2$ with $\mathrm{p} 53$ contributes to the development of HD as well. Alzheimer's disease (AD), acting the characteristics like the progressive decline of memory and cognitive functions as well as changes in behavior and personality. Its mechanism is still quite unclear, but more evidences indicate that ROS and the axon degeneration caused by mitochondrial dysfunction contribute greatly in the process of AD. p53 will transactivate SCO2, NQO1, POX. These factors will increase ROS generation under stress state. Besides, ROS break p53 DNA structure and DNA-binding domain, leading into the expression of unfolded p53, which can be marked as an early marker of oxidative imbalance in AD patients. Accumulation of ROS result in mitochondrial dysfunction, which functions vitally in the process of axon degeneration. Abbreviations: $\mathrm{mtHtt}$ : mutated Huntingtin protein, Drp1: dynamin-related protein 1, NQO1: quinone oxidoreductase or $\mathrm{NAD}(\mathrm{P}) \mathrm{H}$ dehydrogenase, quinone 1, POX: proline oxidase 1

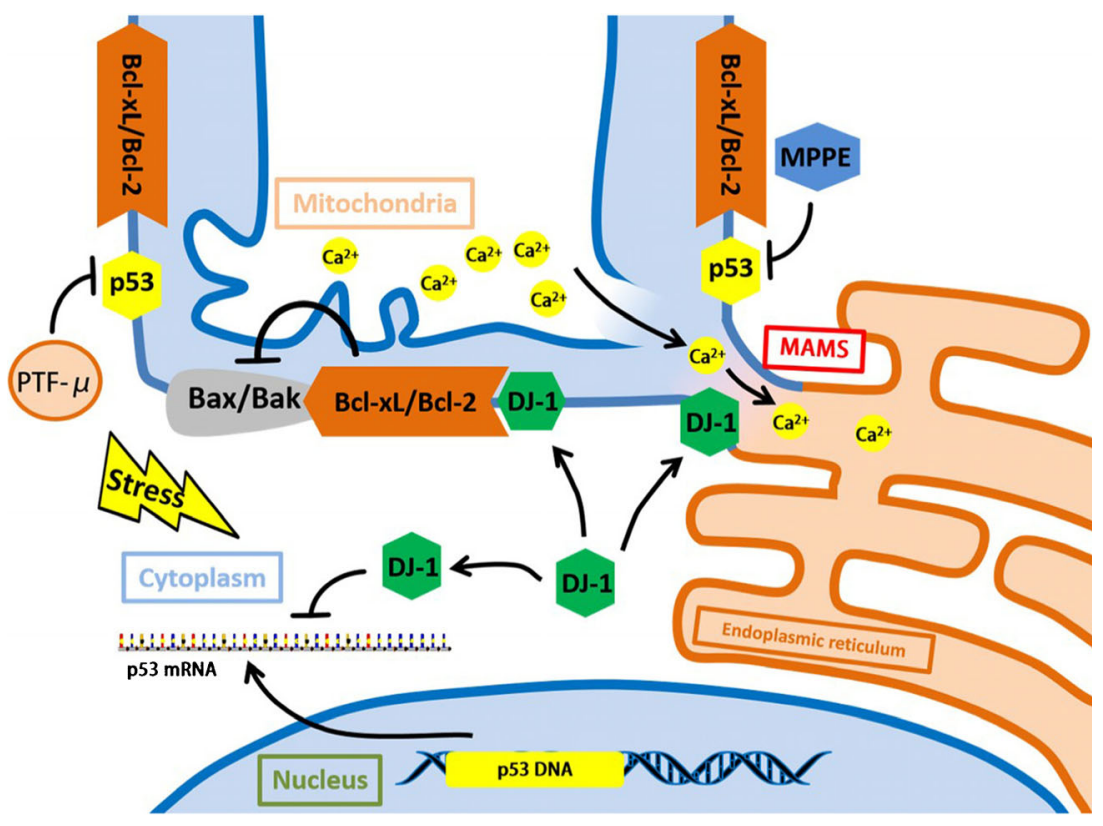

Fig. 4 Protective molecules and pathways in PD. DJ-1 is the Parkinson disease-related protein. Its over overexpression reduces $\mathrm{p} 53$ expression in transactivation and mRNA levels. The presence of DJ-1 greatly stabilizes Mitochondrial Associated ER Membranes (MAMs) and enhances the $\mathrm{Ca}^{2+}$ cross-talk between the two organelles. Alongside this, DJ-1 can bind and stabilize the anti-apoptotic protein $\mathrm{Bcl}-\mathrm{xL}$, permitting its inhibition towards Bax/Bak. PTF- $\mu$, MPPE, Mn-SOD can inhibit p53 mitochondrial translocation and the interaction of $\mathrm{p} 53 / \mathrm{Bcl}-\mathrm{xL}$. Abbreviations: MAMs:Mitochondrial Associated ER Membranes, PTF$\mu$ : pifithrin- $\mu$, MPPE: N-Methyl, N-propynyl-2-phenylethylamine, MnSOD:mitochondrial superoxide dismutase 
indicates that mitochondrial dysfunction caused by $\mathrm{p} 53$ overexpression is the important factor to develop PD. (Fig.3).

In the aspect of therapy, we utilize some molecules and pathways to put forward four treating methods of Parkinson's disease. (Fig.4).

\section{DJ-1 overexpression in neuroprotection}

DJ-1 is the Parkinson disease-related protein, its overexpression overcomes p53-induced mitochondrial phenotypes, suggesting that the protective DJ-1 role may be related to the control of p53 turnover. Its function towards PD can be totally divided in to three parts.

Under the stress state, DJ-1 overoverexpression reduces p53 expression in transactivation and mRNA levels. Moreover, the presence of DJ-1 greatly stabilizes Mitochondrial Associated ER Membranes (MAMs) and enhances the $\mathrm{Ca}^{2+}$ cross-talk between the two organelles. Alongside this, DJ-1 can bind and stabilize the antiapoptotic protein $\mathrm{Bcl}-\mathrm{xL}$, permitting its inhibition towards Bax/Bak.(Ottolini et al. 2013).

\section{Pifithrin- $\mu$ in neuroprotection}

As we discussed previously, p53/Bcl-xL interaction can impair mitochondrial membrane potential and balance, contributing greatly to the process of apoptosis and reducing some pathological changes in Parkinson's disease, Pifithrin- $\mu$ can effectively block p53 form its combining to Bcl-xL (Shin et al. 2015).

\section{N-methyl, N-propynyl-2-phenylethylamine (MPPE) in neuroprotection}

In PD patients, one of the factors inducing dopaminergic toxicity is 1-methyl-4-phenyl-1,2,3,6-tetrahydropyridine. EunJoo Shin et al. demonstrated that N-Methyl, N-propynyl-2phenylethylamine (MPPE) can inhibit p53 mitochondrial translocation and the interaction of $\mathrm{p} 53 / \mathrm{Bcl}-\mathrm{xL}$ in this dopaminergic toxicity. (Shin et al. 2015).

\section{Mn-SOD in neuroprotection}

Moreover, p53 can bind to mitochondrial Mn-SOD (SOD-2), leading to the suppression of its superoxide scavenging activity (Zhao et al. 2005). By upregulating mitochondrial MnSOD expression and UCP-2 mRNA expression, p53 toxicity can be alleviated greatly in Parkinson's disease.

\section{Huntington's disease}

Huntington's disease (HD) is caused by the expansion of the coding CAG repeat in the open reading frame of the
Huntingtin gene The result of accumulation and expression of mutated Huntingtin protein ( $\mathrm{mtHtt}$ ) will lead to cellular dysfunctions, thus, causing HD (Guo et al. 2013). Besides, recent studies revealed that p53 contributes the mitochondria-associated cellular dysfunction and behavioral abnormalities in HD (Bae et al. 2005).

$\mathrm{mtHtt}$ with expanded polyglutamine (polyQ) binds to $\mathrm{p} 53$ and upregulates nuclear p53 level as well as p53 transcriptional activity (Bae et al. 2005). We summarize two main pathways in overexpression of p53 contributing to the development of HD.

The first is that $\mathrm{mtHtt}$ can change the balance between mitochondrial fusion and fission by regulating p53 and Drp1.With the help of mtHtt, p53 binds to activated Drp1 under the stress state, which greatly enhance the interaction of Drp1 and Fis1 on mitochondrial outer membrane, causing the mitochondrial fission (Guo et al. 2013, Schapira et al. 2014). Moreover, mtHtt disturbs interaction between p53 with MDM2, which stabilizes p53 and decreases the p53 degeneration (Bae et al. 2005, Tsoi and Chan 2013).

Besides, the interaction of Bcl-xL/Bcl-2 with $\mathrm{p} 53$ still contributes to the development of HD. (Fig.3).

Here are two possible treating methods of HD.

\section{Drp1 and p110}

Xing Guo et al. demonstrated that by binding to Drp1 and mediating Drp1-induced mitochondrial and neuronal damage, p53 contributes greatly to the development of HD pathology.

As previously discussed, p110 can inhibit necrosis. Moreover, Xing Guo et al. revealed that with suppressing $\mathrm{mtHtt}-$ induced association of $\mathrm{p} 53$ with mitochondria and mitochondrial morphology, p110 can reduce $\mathrm{mtHtt}$-induced excessive mitochondrial fragmentation, thus, improving mitochondrial function and cell viability in HD cell culture models (Guo et al. 2013).

Most importantly, p110 can block the interaction between Drp1/p53 complex and Fis1, alleviating the mitochondrial fission, and suppress the interaction of $\mathrm{p} 53$ and Bcl-xl or Bcl-2, protecting cells from apoptosis (Guo et al. 2013). (Fig. 5).

\section{Human adipose stem cells (hASC) extracts}

in in neuroprotection

hASC is confirmed to be able to produce many essential neurotrophic factors in neuronal growth and survival. Tian Liu et al. revealed that hASC extracts can prevent mHtt-induced apoptosis, alleviate mHtt-induced mitochondrial oxidative stress and recover mitochondrial membrane potential from reduction. Interestingly, hASC extracts can also block the interaction of p53 and $\mathrm{mHtt}$, and decrease half-life of the endogenous 


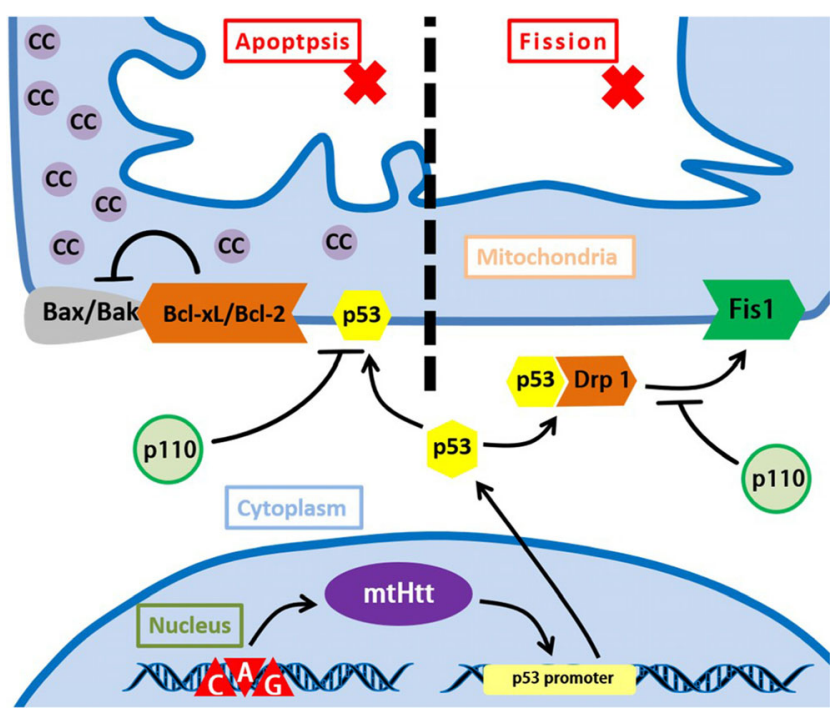

Fig. 5 p110 protective role in HD. p110 can block the interaction between Drp1/p53 complex and Fis1, alleviating the mitochondrial fission and suppress the interaction of $\mathrm{p} 53$ and $\mathrm{Bcl}-\mathrm{xl}$ or Bcl-2, protecting cells from apoptosis

p53(Liu et al. 2014). However, the exact molecule and pathway of these effects needs to be further studied.

\section{Alzheimer's disease}

Alzheimer's disease (AD), acting the characters like the progressive decline of memory and cognitive functions as well as changes in behavior and personality (Manczak et al. 2013). Its mechanism is still quite unclear, but more evidences indicate that ROS and the axon degeneration caused by mitochondrial dysfunction contribute greatly in the process of AD (Fiorini et al. 2012, Fujiwara and Morimoto 2012, 2013). Moreover, Laura Buizza et al. confirmed that ROS/RNS contribute to variation of p53 structure and that unfolded p53 can be marked as an early marker of oxidative imbalance in $\mathrm{AD}$ patients (Buizza et al. 2012).

Besides, more evidences suggested impaired mitochondrial function has been shown in Alzheimer's disease patients as well as animal and cell models of AD (Hedskog et al. 2013). In the central nervous system of AD patients, increasing evidence indicates that mitochondrial dysfunction occurs in brain (Mancuso et al. 2003). Decreasing of the mitochondrial cytochrome c oxidase (COX) activity is the significant performance (Mancuso et al. 2003). Besides, J. Casademont et al. also demonstrated that defects in mitochondria like the dysfunction of electron transport chain (ETC), might play the vital role in the $\mathrm{AD}$ pathological changes. It has been found that ETC is decreased in AD brain (Casademont et al. 2003).

All these results indicated the correlation of $\mathrm{p} 53$ and mitochondria in AD development. (Fig.3).

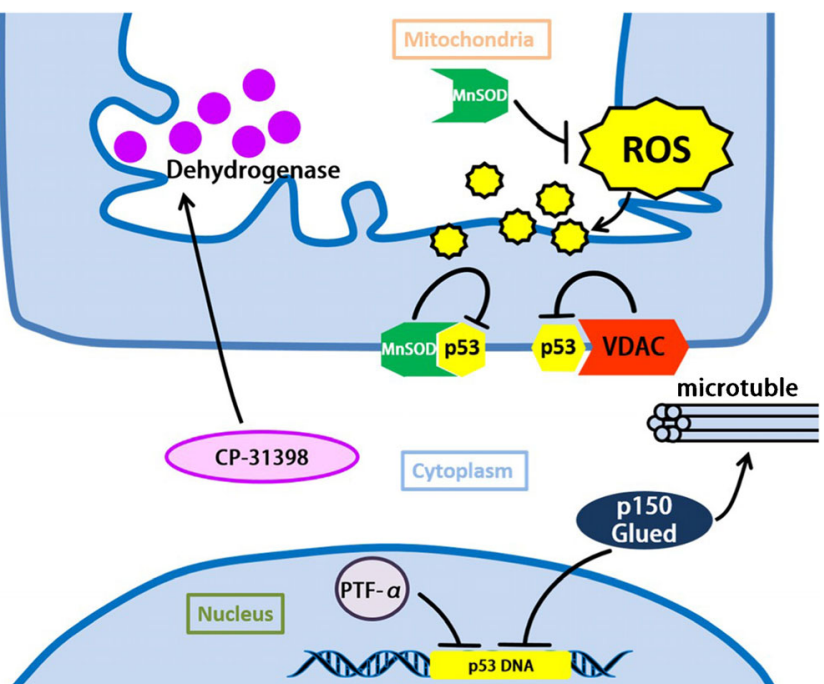

Fig. 6 Protective molecules and pathways in AD. Mn-SOD contributes as the anti-oxidative factor and is involved in the cellular defense against oxidative damage caused by ROS. Overexpression of MnSOD can inhibit p53 from combining with other protein like Bcl-2. VDAC is found to be connected with restraining p53 under the stress state. CP-31398 is a known compound which can rescue the p53 mutant structure and stabilize the activation of wild-type form. Besides, CP-31,398 can suppress mitochondrial dysfunction by increasing mitochondrial dehydrogenase activity. PFT- $\alpha$ can inhibit p53 transactivation, and result in the suppression of axon degeneration. p150Glued can inhibit p53 transactivation and stabilize microtubule, which modulates mitochondria function and improves axons integrity to avoid excitotoxicity-induced degeneration. Abbreviations: VDAC: voltagedependent anion channel

On the aspect of treatment, we summarize five possible targeted molecular therapies towards Alzheimer's disease. (Fig.6).

\section{Mitochondrial superoxide dismutase (MnSOD) in neuroprotection}

MnSOD contributes as the anti-oxidative factor and is involved in the cellular defense against oxidative damage caused by ROS (Sharma et al. 2016).

Alongside this function, Ada Fiorini et al. revealed that overexpression of Mn-SOD can protect neurons against oxidative damage (Fujiwara and Morimoto 2012). Overexpression of MnSOD can inhibit 553 from combining with other protein like Bcl-2, which is helpful to prevent or slow down the progression of $\mathrm{AD}$ processes (Fiorini et al. 2012).

\section{Voltage-dependent anion channel (VDAC) in neuroprotection}

Maria Manczak et al. suggested that reduced voltagedependent anion channel 1 (VDAC1) expression may be beneficial for synaptic for activity. VDAC is one of the components in mitochondria permeability transition pore (MPTP), the upregulation of VDAC contributes to the balance and 
improvement of synaptic transmission and cell survival (Fiorini et al. 2012),thus, improving function as well as protecting against toxicities of AD-related genes (Manczak et al. 2013).

Moreover, VDAC is found to be connected with p53, like restraining p53 under the stress state (Fiorini et al. 2012).

\section{CP-31,398 in neuroprotection}

It has been demonstrated that glutamate excitotoxicity is connected with the development in AD. Takeshi Fujiwara et al. found that CP-31,398 can suppress glutamate excitotoxicity-induced axon degeneration. Interestingly, CP-31,398 is a known compound which can rescue the p53 mutant structure and stabilize the activation of wild-type form. Besides, Takeshi Fujiwara et al. demonstrated that CP-31,398 can suppress mitochondrial dysfunction by increasing mitochondrial dehydrogenase activity compared with glutamate treatment. However, the exact interaction and pathway of CP31,398 toward this effect needs further study afterwards. (Fujiwara and Morimoto 2013).

\section{PFT- $\alpha$ in neuroprotection}

As we discussed previously, p53 transactivation towards Bax, PUMA, and NOXA may lead to apoptosis. Takeshi Fujiwara et al. found that $\mathrm{p} 53$ transactivation can be inhibited by PFT- $\alpha$, resulting in the suppression of axon degeneration, and this treatment can be more efficient when treated together with taxol. (Fujiwara and Morimoto 2012).

\section{p150Glued in neuroprotection}

p150Glued is a major component of the dynactin complex. Takeshi Fujiwara et al. found out that overexpression of p150Glued can inhibit p53 transactivation and stabilize microtubule, which modulates mitochondria function and improve axons integrity to avoid excitotoxicity-induced degeneration (Fujiwara and Morimoto 2012).

\section{Outlook of treatment}

Neuronal diseases are common but hard to cure. But we still hold the positive opinion towards common treating method suggested by Anthony H V Schapira et al., which means ablating expression, counteracting with antibody fragments, enhancing the clearance towards mutant or unwanted protein and correcting the abnormal conditions. (Schapira et al. 2014) These four treating methods might guide us into the recovery of the neurodegenerative diseases. In this review, the molecular therapies towards p53 and mitochondria can be classified into the four methods mentioned above, like ablating p53 expression by PFT- $\alpha$ in AD treating or correcting the apoptosis condition by p110 in HD treating. We expected that these novel molecular therapies can function effectively in future clinic.

However, there is still a problem need to be resolved, which is the opposite function of p53 in different cells under different stress levels. As we can see in Fig. 1a, in normal condition, p53 can maintain aerobic respiratory through SCO2(Fields et al. 2007). Moreover, p53 can transactivate Mieap to degenerate oxidized protein to protect cells (Miyamoto et al. 2011). But with enhancement of the stress levels, p53 will lead to the mitochondrial dysfunction and finally apoptosis.

In the process of cell aging, p53 will be overexpressed to against DNA mutation or the Warburg effect (Matoba et al. 2006). p53 can take the protective role by keeping or enhancing aerobic respiratory through $\mathrm{SCO} 2$ and Mieap expression to degenerate oxidized protein under this condition. However, when high level stress presents, p53 overexpression functions as a detrimental role in nervous system especially in the neurons related of neurodegenerative diseases, which aggravates the symptoms of these diseases. The rank of the stress level can then be the vital factor in the therapies towards p53 and mitochondria, through which we can determine when to increase $\mathrm{p} 53$ for protecting cell or degenerate p53 for inhibiting damage. The exact stress level rank and clear definition of p53 function changing needs further study.

We look forward to more new and efficient treating therapies from the aspect of p53 and mitochondria.

Acknowledgments This work was supported by grants from the National Natural Science Foundation of China $(81272555,81470843)$.

\section{Compliance with ethical standards}

Conflict of interest The authors have no conflict of interest.

\section{References}

Assaily W, Benchimol S (2006) Differential utilization of two ATPgenerating pathways is regulated by $\mathrm{p} 53$. Cancer Cell 10:4-6

Bae BI, Xu H, Igarashi S, Fujimuro M, Agrawal N, Taya Y, Hayward SD, Moran TH, Montell C, Ross CA, Snyder SH, Sawa A (2005) p53 mediates cellular dysfunction and behavioral abnormalities in Huntington's disease. Neuron 47:29-41

Bergeaud M, Mathieu L, Guillaume A, Moll UM, Mignotte B, Le Floch N, Vayssiere JL, Rincheval V (2013) Mitochondrial p53 mediates a 
transcription-independent regulation of cell respiration and interacts with the mitochondrial F(1)F0-ATP synthase. Cell Cycle 12:27812793

Buizza L, Cenini G, Lanni C, Ferrari-Toninelli G, Prandelli C, Govoni S, Buoso E, Racchi M, Barcikowska M, Styczynska M, Szybinska A, Butterfield DA, Memo M, Uberti D (2012) Conformational altered p53 as an early marker of oxidative stress in Alzheimer's disease. PLoS One 7:e29789

Casademont J, Miro O, Rodriguez-Santiago B, Viedma P, Blesa R, Cardellach F (2003) Cholinesterase inhibitor rivastigmine enhance the mitochondrial electron transport chain in lymphocytes of patients with Alzheimer's disease. J Neurol Sci 206:23-26

Chen F, Wang W, El-Deiry WS (2010) Current strategies to target p53 in cancer. Biochem Pharmacol 80:724-730

Dashzeveg N, Yoshida K (2015) Cell death decision by p53 via control of the mitochondrial membrane. Cancer Lett 367:108-112

Erster S, Mihara M, Kim RH, Petrenko O, Moll UM (2004) In vivo mitochondrial $\mathrm{p} 53$ translocation triggers a rapid first wave of cell death in response to DNA damage that can precede p53 target gene activation. Mol Cell Biol 24:6728-6741

Esposito F, Tornincasa M, Federico A, Chiappetta G, Pierantoni GM, Fusco A (2012) High-mobility group A1 protein inhibits p53mediated intrinsic apoptosis by interacting with Bcl-2 at mitochondria. Cell Death Dis 3:e383

Feng J, Meng C, Xing D (2015) Abeta induces PUMA activation: a new mechanism for Abeta-mediated neuronal apoptosis. Neurobiol Aging 36:789-800

Fields J, Hanisch JJ, Choi JW, Hwang PM (2007) How does p53 regulate mitochondrial respiration? IUBMB life 59:682-684

Fiorini A, Sultana R, Barone E, Cenini G, Perluigi M, Mancuso C, Cai J, Klein JB, St Clair D, Butterfield DA (2012) Lack of p53 affects the expression of several brain mitochondrial proteins: insights from proteomics into important pathways regulated by $\mathrm{p} 53$. PLoS One 7:e49846

Fujiwara T, Morimoto K (2012) Inhibition of p53 transactivation functionally interacts with microtubule stabilization to suppress excitotoxicity-induced axon degeneration. Biochem Biophys Res Commun 427:100-106

Fujiwara T, Morimoto K (2013) A compound CP-31398 suppresses excitotoxicity-induced neurodegeneration. Biochem Biophys Res Commun 440:359-363

Gibellini L, Bianchini E, De Biasi S, Nasi M, Cossarizza A, Pinti M (2015) Natural compounds modulating mitochondrial functions. Evidence-based complementary and alternative medicine: eCAM 2015:527209

Guo X, Disatnik MH, Monbureau M, Shamloo M, Mochly-Rosen D, Qi $X$ (2013) Inhibition of mitochondrial fragmentation diminishes Huntington's disease-associated neurodegeneration. J Clin Invest 123:5371-5388

Guo X, Sesaki H, Qi X (2014) Drp1 stabilizes p53 on the mitochondria to trigger necrosis under oxidative stress conditions in vitro and in vivo. The Biochemical journal 461:137-146

Hang L, Thundyil J, Lim KL (2015) Mitochondrial dysfunction and Parkinson disease: a Parkin-AMPK alliance in neuroprotection. Ann N Y Acad Sci 1350:37-47

Hedskog L, Pinho CM, Filadi R, Ronnback A, Hertwig L, Wiehager B, Larssen P, Gellhaar S, Sandebring A, Westerlund M, Graff C, Winblad B, Galter D, Behbahani H, Pizzo P, Glaser E, Ankarcrona M (2013) Modulation of the endoplasmic reticulum-mitochondria interface in Alzheimer's disease and related models. Proc Natl Acad Sci U S A 110:7916-7921

Hernlund E, Kutuk O, Basaga H, Linder S, Panaretakis T, Shoshan M (2009) Cisplatin-induced nitrosylation of $\mathrm{p} 53$ prevents its mitochondrial translocation. Free Radic Biol Med 46:1607-1613

Kamino H, Nakamura Y, Tsuneki M, Sano H, Miyamoto Y, Kitamura N, Futamura M, Kanai Y, Taniguchi H, Shida D, Kanemitsu Y, Moriya
Y, Yoshida K, Arakawa H (2016) Mieap-regulated mitochondrial quality control is frequently inactivated in human colorectal cancer. Oncogenesis 4:e181

Kitamura N, Nakamura Y, Miyamoto Y, Miyamoto T, Kabu K, Yoshida M, Futamura M, Ichinose S, Arakawa H (2011) Mieap, a p53inducible protein, controls mitochondrial quality by repairing or eliminating unhealthy mitochondria. PLoS One 6:e16060

Lee SK, Kim YS (2013) Phosphorylation of eIF2alpha attenuates statininduced apoptosis by inhibiting the stabilization and translocation of p53 to the mitochondria. Int J Oncol 42:810-816

Lee JY, Jee SB, Park WY, Choi YJ, Kim B, Kim YH, do Y J, Kim YH (2014) Tumor suppressor protein $\mathrm{p} 53$ promotes 2-methoxyestradiolinduced activation of Bak and Bax, leading to mitochondriadependent apoptosis in human colon cancer HCT116 cells. J Microbiol Biotechnol 24:1654-1663

Liu T, Im W, Lee ST, Ban JJ, Chai YJ, Lee M, Mook-Jung I, Chu K, Kim M (2014) Modulation of mitochondrial function by stem cellderived cellular components. Biochem Biophys Res Commun 448:403-408

Madan E, Gogna R, Kuppusamy P, Bhatt M, Mahdi AA, Pati U (2013) SCO2 induces p53-mediated apoptosis by Thr845 phosphorylation of ASK-1 and dissociation of the ASK-1-Trx complex. Mol Cell Biol 33:1285-1302

Mancuso M, Filosto M, Bosetti F, Ceravolo R, Rocchi A, Tognoni G, Manca ML, Solaini G, Siciliano G, Murri L (2003) Decreased platelet cytochrome c oxidase activity is accompanied by increased blood lactate concentration during exercise in patients with Alzheimer disease. Exp Neurol 182:421-426

Manczak M, Sheiko T, Craigen WJ, Reddy PH (2013) Reduced VDAC1 protects against Alzheimer's disease, mitochondria, and synaptic deficiencies. Journal of Alzheimer's disease: JAD 37:679-690

Matoba S, Kang JG, Patino WD, Wragg A, Boehm M, Gavrilova O, Hurley PJ, Bunz F, Hwang PM (2006) p53 regulates mitochondrial respiration. Science 312:1650-1653

Miyamoto Y, Kitamura N, Nakamura Y, Futamura M, Miyamoto T, Yoshida M, Ono M, Ichinose S, Arakawa H (2011) Possible existence of lysosome-like organella within mitochondria and its role in mitochondrial quality control. PLoS One 6:e16054

Miyamoto T, Kitamura N, Ono M, Nakamura Y, Yoshida M, Kamino H, Murai R, Yamada T, Arakawa H (2012) Identification of 14-33 gamma as a Mieap-interacting protein and its role in mitochondrial quality control. Scientific reports 2:379

Ottolini D, Cali T, Negro A, Brini M (2013) The Parkinson diseaserelated protein DJ-1 counteracts mitochondrial impairment induced by the tumour suppressor protein $\mathrm{p} 53$ by enhancing endoplasmic reticulum-mitochondria tethering. Hum Mol Genet 22:2152-2168

Park JY, Choi H, Baek S, Jang J, Lee A, Jeon S, Kim J, Park HJ (2015) p53 signalling mediates acupuncture-induced neuroprotection in Parkinson's disease. Biochem Biophys Res Commun 460:772-779

Qi Z, He J, Su Y, He Q, Liu J, Yu L, Al-Attas O, Hussain T, Ding S, Ji L, Qian M (2011) Physical exercise regulates p53 activity targeting $\mathrm{SCO} 2$ and increases mitochondrial COX biogenesis in cardiac muscle with age. PLoS One 6:e21140

Saleem A, Hood DA (2013) Acute exercise induces tumour suppressor protein $\mathrm{p} 53$ translocation to the mitochondria and promotes a $\mathrm{p} 53$ Tfam-mitochondrial DNA complex in skeletal muscle. J Physiol 591:3625-3636

Sansome C, Zaika A, Marchenko ND, Moll UM (2001) Hypoxia death stimulus induces translocation of $\mathrm{p} 53$ protein to mitochondria. Detection by immunofluorescence on whole cells FEBS letters 488:110-115

Schapira AH, Olanow CW, Greenamyre JT, Bezard E (2014) Slowing of neurodegeneration in Parkinson's disease and Huntington's disease: future therapeutic perspectives. Lancet 384:545-555

Seo YW, Shin JN, Ko KH, Cha JH, Park JY, Lee BR, Yun CW, Kim YM, Seol DW, Kim DW, Yin XM, Kim TH (2003) The molecular 
mechanism of Noxa-induced mitochondrial dysfunction in p53mediated cell death. J Biol Chem 278:48292-48299

Sharma DR, Wani WY, Sunkaria A, Kandimalla RJ, Sharma RK, Verma D, Bal A, Gill KD (2016) Quercetin attenuates neuronal death against aluminum-induced neurodegeneration in the rat hippocampus. Neuroscience 324:163-176

Shin EJ, Nam Y, Lee JW, Nguyen PT, Yoo JE, Tran TV, Jeong JH, Jang CG, Oh YJ, Youdim MB, Lee PH, Nabeshima T, Kim HC (2015) Nmethyl, N-propynyl-2-phenylethylamine (MPPE), a Selegiline ana$\log$, attenuates MPTP-induced dopaminergic toxicity with guaranteed behavioral safety: involvement of inhibitions of mitochondrial oxidative burdens and p53 Gene-elicited pro-apoptotic change. Molecular neurobiology.

Tsoi H, Chan HY (2013) Expression of expanded CAG transcripts triggers nucleolar stress in Huntington's disease. Cerebellum 12:310-312

Tsuneki M, Nakamura Y, Kinjo T, Nakanishi R, Arakawa H (2015) Mieap suppresses murine intestinal tumor via its mitochondrial quality control. Scientific reports 5:12472

Vaseva AV, Moll UM (2009) The mitochondrial p53 pathway. Biochim Biophys Acta 1787:414-420
Wan C, Ma X, Shi S, Zhao J, Nie X, Han J, Xiao J, Wang X, Jiang S, Jiang J (2014) Pivotal roles of p53 transcriptiondependent and -independent pathways in manganese-induced mitochondrial dysfunction and neuronal apoptosis. Toxicol Appl Pharmacol 281:294-302

Wang F, Fu X, Chen X, Chen X, Zhao Y (2010) Mitochondrial uncoupling inhibits p53 mitochondrial translocation in TPAchallenged skin epidermal JB6 cells. PLoS One 5:e13459

Wang DB, Kinoshita C, Kinoshita Y, Morrison RS (2014) p53 and mitochondrial function in neurons. Biochim Biophys Acta 1842:1186-1197

Yang H, Brosel S, Acin-Perez R, Slavkovich V, Nishino I, Khan R, Goldberg IJ, Graziano J, Manfredi G, Schon EA (2010) Analysis of mouse models of cytochrome c oxidase deficiency owing to mutations in Sco2. Hum Mol Genet 19: 170-180

Zhao Y, Chaiswing L, Velez JM, Batinic-Haberle I, Colburn NH, Oberley TD, St Clair DK (2005) p53 translocation to mitochondria precedes its nuclear translocation and targets mitochondrial oxidative defense protein-manganese superoxide dismutase. Cancer Res 65:3745-3750 